\title{
Assessment of the relationship between hepatocellular carcinoma location and its response to transarterial chemoembolization
}

\author{
Ahmed Elsahhar * (1), Sameh M. Abdelwahab, Haytham M. Nasser and Mohammed Sobhi Hassan
}

\begin{abstract}
Background: Transarterial chemoembolization (TACE) is the recommended treatment in intermediate stage of hepatocellular carcinoma (HCC). Many indices are used to predict the outcome of the TACE. The location of the HCC has not been enough studied as a prognostic variable.

Results: We evaluated 149 HCC nodules (111 patients) and analyzed the association between the response to TACE and the nodule location. There was a significant difference between the complete response (CR) and the non-CR group in the location of the HCC regarding its segment and the response to TACE with the anterior segment lesions achieving higher rates of complete response (CR) ( $P$.03), and the distance between the tumor and the liver capsule with the subcapsular lesions showing higher rate of non-CR ( $P$.02). However, there was no significant difference between the $C R$ and the non-CR groups regarding the location between the right and the left lobes $(P .48)$ and the central versus peripheral lesions ( $P .41)$.

Conclusions: The location of the HCC can improve the prediction of the tumor response to TACE. Anterior segment tumors showed a higher rate of complete response and subcapsular lesions showed a higher rate of recurrence after TACE.
\end{abstract}

Keywords: Hepatocellular carcinoma, Transarterial chemoembolization, Lipidol, Cirrhosis

\section{Background}

Primary liver cancer is the sixth most common malignancy worldwide. Hepatocellular carcinoma (HCC) represents $75-85 \%$ of those cases [1]. Transarterial chemoembolization (TACE) is recommended in management of stage B patients of Barcelona Clinic liver Cancer (BCLC) staging system. TACE combines delivering chemotherapeutic agents to the tumor as well as arterial embolization to induce tumor necrosis [2].

Liver cirrhosis induces morphometric and perfusion alterations in the liver parenchyma. These changes are more prominent in Child-Pugh Class A patients and decrease with the progression of the disease [3].

A study on hepatitis C-related cirrhotic patients demonstrated anterior and middle segments atrophy with lateral and posterior segments and caudate lobe hypertrophy. This was attributed to higher venous pressure in the middle hepatic vein drainage area due to compression caused by cirrhotic nodules [4]. Also, a study showed that in case of portal vein obstruction there is a decrease in peripheral zone perfusion with atrophic changes, whereas the central zone acquires more blood flow causing its hypertrophy [5].

${ }^{*}$ Correspondence: ahmed.elsahhar@med.asu.edu.eg

Department of Radiology, Ain Shams University, Cairo, Egypt 


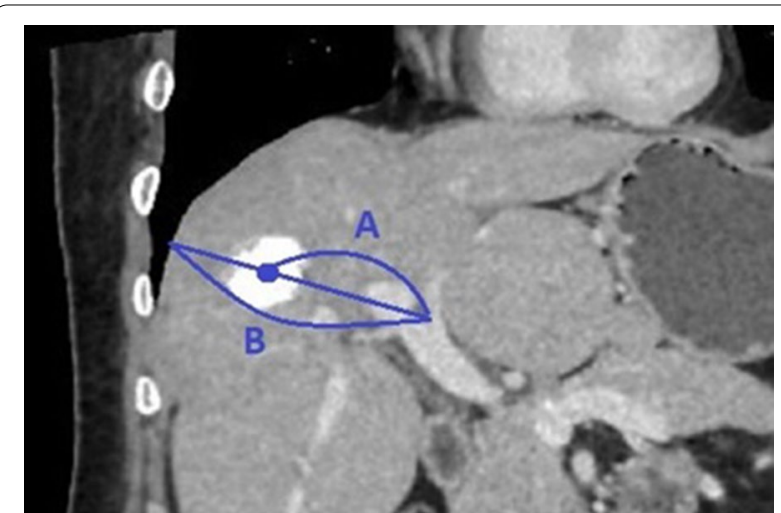

Fig. 1 The coefficient of the HCC = distance from the hilum of the liver at the portal vein bifurcation to the center of the HCC $(\mathbf{A})$ divided by the diameter of the whole liver on the same line (B)

The purpose of this study was to assess the relationship between the location of the HCC and its response to TACE.

\section{Methods \\ Patients}

Retrospective analysis of patients who underwent TACE in our institution between January 2019 and December 2020 was performed. Inclusion criteria were as follows: (1) diagnosis of HCC by CT, MRI or histopathology, (2) BCLC stage B patients, (3) patients who had naïve lesions, (4) patients who underwent conventional TACE and (5) technical success of TACE. Exclusion criteria were as follows: (1) recurrent HCC, (2) nodules who underwent additional ablation with TACE and (3) the presence of vascular invasion. Our study included 149 nodules (111 patients).

\section{TACE procedure}

Puncture of the right common femoral artery and inserting $6 \mathrm{~F}$ vascular sheath was performed. A $5 \mathrm{~F}$ Cobra head catheter is introduced over $0.035^{\prime \prime}$ hydrophilic guidewire, or coaxial microcatheter if necessary, and celiac-mesenteric angiography is done to confirm feeding arteries to HCC and assess liver anatomy. Selective catheterization was done as distal as possible to minimize collateral liver injury but proximal enough to treat the entire targeted lesion. Delivery of chemotherapy doxorubicin $50 \mathrm{mg} / \mathrm{m}^{2}$ (up to $100 \mathrm{mg}$ ) emulsified in Lipiodol under continuous fluoroscopic visualization followed by gelfoam embolization.

\section{HCC location}

The location of the HCC was determined according to right and left lobes and anterior, posterior, lateral and medial segments and according to the Couinaud classification. Also, the location being central versus peripheral was defined by the coefficient of the HCC, which is the ratio of the distance from the hilum of the liver at the portal vein bifurcation to the center of the HCC (A) divided by the diameter of the whole liver on the same line (B) (Fig. 1.). Higher coefficient means more peripheral location, and lower coefficient means more central location.

\section{Evaluation of treatment response}

Contrast-enhanced CT or MRI (1 month after TACE) was used for follow-up and mRECIST criteria were used to assess tumor response [6] (Table 1).

\section{Statistical analysis}

The Mann-Whitney U test, Kruskal-Wallis test and Chisquare test were used to assess the association between response to TACE and different variables. All analyses were performed using SPSS software version 26.0. A P value of $<0.05$ was considered significant.

\section{Results}

TACE procedures were done on 149 nodules (111 patients). The baseline characteristics are summarized in Table 2.

The response to TACE was classified to complete response (CR) group (Fig. 2) and non-CR group (Fig. 3) including partial response (PR), stable disease (SD) and progressive disease (PD). Results are summarized in Table 3 and the response in each lobe and segment is summarized in Table 4.

Table 1 mRECIST criteria

\begin{tabular}{ll}
\hline Complete response (CR) & Disappearance of any intratumoral arterial enhancement in all target lesions \\
Partial response (PR) & At least a $30 \%$ decrease in the sum of diameters of viable target lesions \\
Stable disease (SD) & Any cases that do not qualify for either partial response or progressive disease \\
Progressive disease (PD) & Increase of at least 20\% in the sum of the diameters of viable (enhancing) target lesions \\
\hline
\end{tabular}


Table 2 Baseline characteristics

\begin{tabular}{lll}
\hline Age (years) & Mean \pm standard deviation & $61.7 \pm 7.5$ \\
Gender & Median (minimum-maximum) & $62(48-80)$ \\
& Male & $119(79.9 \%)$ \\
Etiology & Female & $30(20.1 \%)$ \\
& Hepatitis C & $144(96.6 \%)$ \\
Child-Pugh score & Hepatitis B & $5(3.4 \%)$ \\
& A & $134(89.9 \%)$ \\
Size of lesions (mm) & B & $15(10.1 \%)$ \\
& Mean \pm standard deviation & $33 \pm 18.6$ \\
Location of the nodules & Median (Minimum-Maximum) & $29(10-95)$ \\
& Anterior segment & $53(35.65)$ \\
& Posterior segment & $52(34.9 \%)$ \\
& Medial segment & $19(12.7 \%)$ \\
& Lateral segment & $21(14.1 \%)$ \\
& Caudate & $4(2.7 \%)$ \\
\hline
\end{tabular}

\section{The relationship between the HCC location and the tumor response}

There was a statistically significant difference between the $C R$ and the non-CR groups regarding the segmental location of the HCC with anterior segment showing higher rates of complete response ( $P$ 0.03) (Fig. 4). However, there was no statistically significant difference between the $C R$ and the non-CR groups regarding the location between the right and the left lobes $(P 0.48)$ (Fig. 5).

As regards the central versus peripheral location of the tumor, there was no statistically significant difference in the median of the location coefficient between the CR and the non-CR groups compared between all segments (P 0.41) (Fig. 6). However, there was a statistically significant difference between the $C R$ and the non-CR groups regarding the distance of the tumor from the capsule of the liver ( $P$ 0.02), with the non-CR group showing shorter distance from the capsule compared to the CR group (Fig. 7).

\section{Discussion}

TACE is the recommended treatment in intermediate stage of HCC [7]. Different prognostic variables are used to predict the efficacy of TACE in different patients including tumor size, portal vein invasion, alpha-fetoprotein level, Child-Pugh grade and performance status [8].

TACE induces vascular endothelial growth factor, which promotes angiogenesis and can cause recurrence in the HCC [9]. Cirrhotic liver has parenchymal and perfusional changes that can alter the response to TACE in different liver regions [5]. Areas with atrophy and decreased perfusion can promote better response to TACE. In this retrospective study, we analyzed the relationship between the location of the $\mathrm{HCC}$ and its response to TACE to assess whether it can be included as a prognostic factor.

A novel finding in our study was the presence of a significant correlation between the segment of the tumor and its response to TACE. The tumors of the anterior segment showed higher complete response rates. A study reported atrophy of the middle hepatic venous drainage area with atrophy of the anterior segment of the liver [4]. In cirrhotic patients, regenerative nodule compresses the hepatic veins and the middle hepatic vein is the most

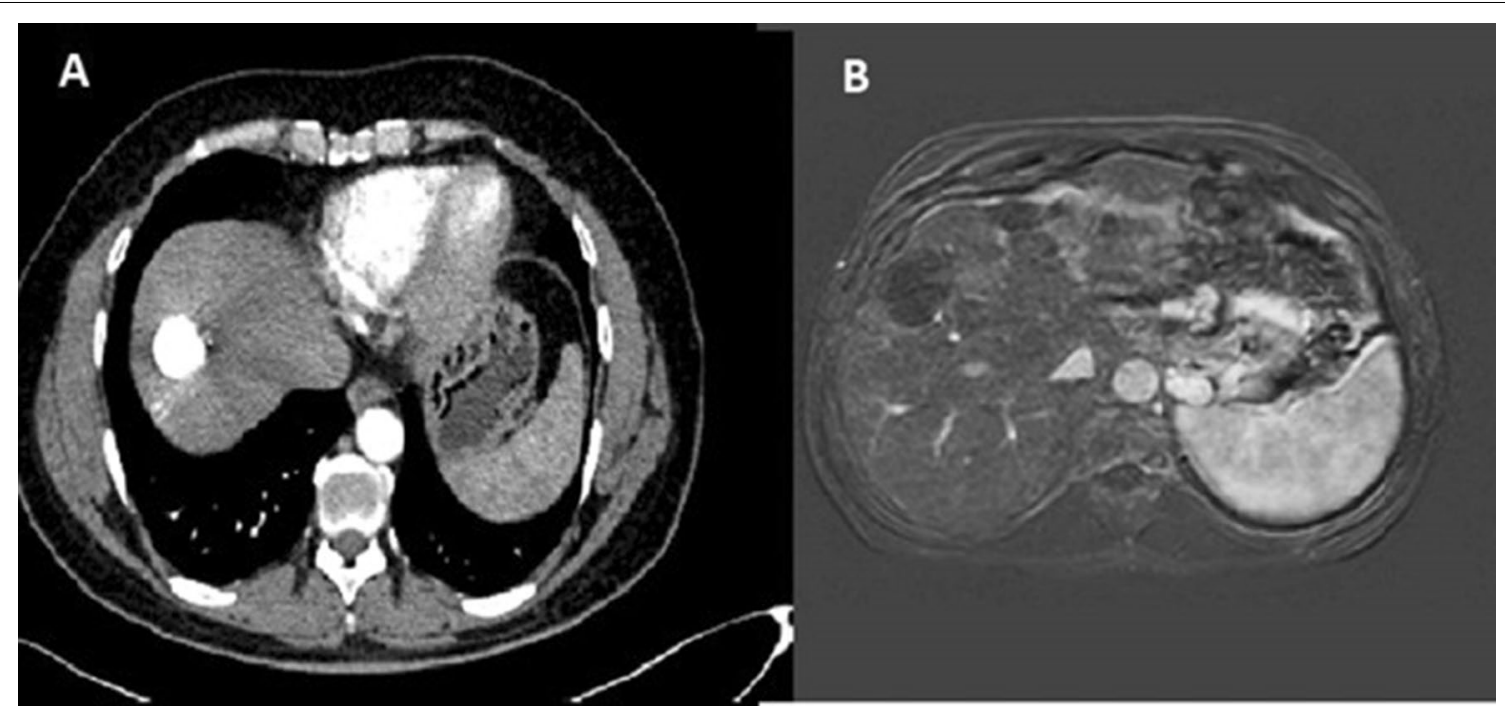

Fig. 2 Complete response: A CT image showing complete Lipiodol deposition with no areas of tumoral arterial enhancement. B MR image showing no intratumoral enhancement 


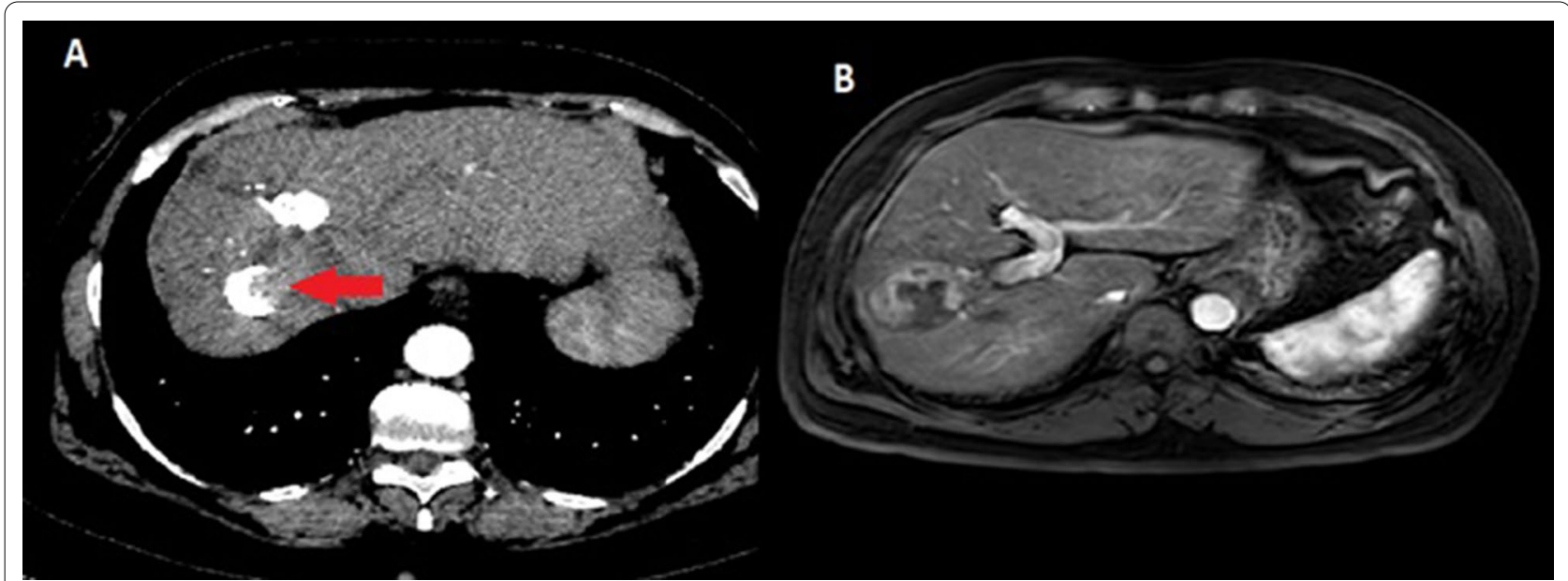

Fig. 3 Non-complete response: A CT (red arrow) and B MR image showing intratumoral arterial enhancement after TACE

Table 3 Response to TACE in all lesions

\begin{tabular}{llll}
\hline Response & CR & & $\mathbf{9 8}(\mathbf{6 5 . 8 \% )}$ \\
\hline & Non-CR & PR & $28(18.8 \%)$ \\
& & SD & $17(11.4 \%)$ \\
& & PD & $6(4 \%)$ \\
\hline
\end{tabular}

Table 4 Response to TACE classified by lobes and segments

\begin{tabular}{lll}
\hline & CR (n) & Non-CR (n) \\
\hline Right lobe & 67 & 38 \\
Left lobe & 28 & 12 \\
Anterior segment & 41 & 12 \\
Posterior segment & 26 & 26 \\
Medial segment & 13 & 6 \\
Lateral segment & 15 & 6 \\
Caudate & 3 & 1 \\
\hline
\end{tabular}

affected one due to its smaller caliber and longer course compared to the other hepatic veins [4].

Several studies showed that peripheral area of the liver had less vascularity compared to the central areas, which should promote better efficacy of TACE in peripheral areas. A retrospective study indicated better response for TACE in the peripheral zone of the right lobe and the medial segment in Child-Pugh grade A patients [10].
However, in our study there was no significant difference between central versus peripheral tumors.

We observed a significant correlation regarding the distance between the tumor and the liver capsule and the efficacy of TACE with non-complete response occurring more frequently in the subcapsular region. Peripherally located and exophytic lesions have higher frequency of having extrahepatic collateral vessels; this can affect the efficacy of TACE [11]. It is suspected when there is a defect in Lipidol retention during the TACE procedure or there is recurrence in the follow-up imaging. Interventional radiologist should be familiar with the possible extrahepatic collateral supply and if possible chemoembolization of those vessels should be done [11].

Our study had several limitations. First, our sample included only HCC with viral etiology. Comparative studies with different etiologies are also necessary. Second, very few lesions were present in the caudate lobe in our study for better assessment of the response to TACE in all the segments.

\section{Conclusions}

The location of the $\mathrm{HCC}$ can improve the prediction of the tumor response to TACE. Anterior segment tumors showed higher rates of complete response, and subcapsular lesions showed a higher rate of recurrence after TACE. 


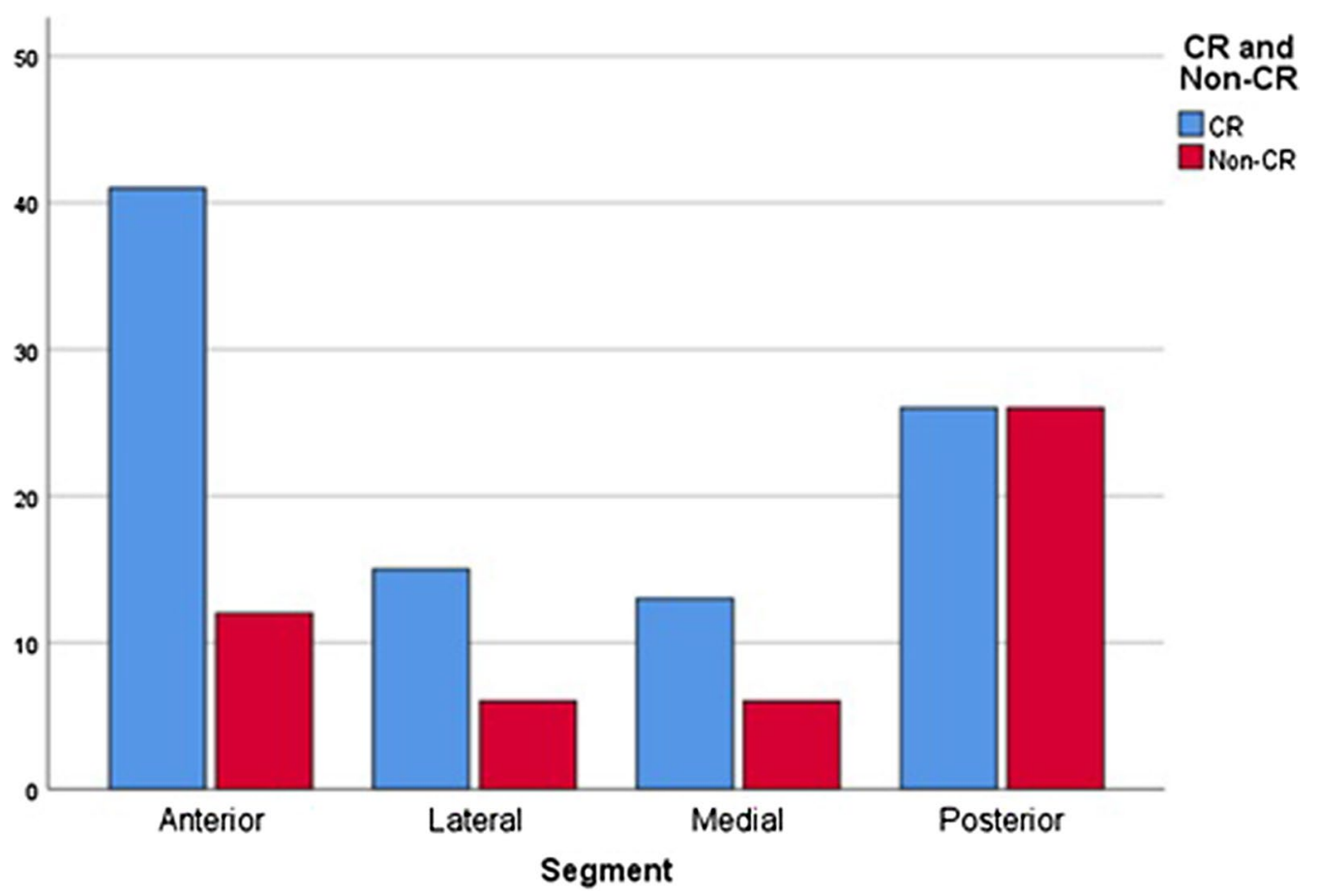

Fig. 4 Bar chart showing the association between the segment of the tumor and $C R$ and non-CR groups, significant difference ( $P$.03)

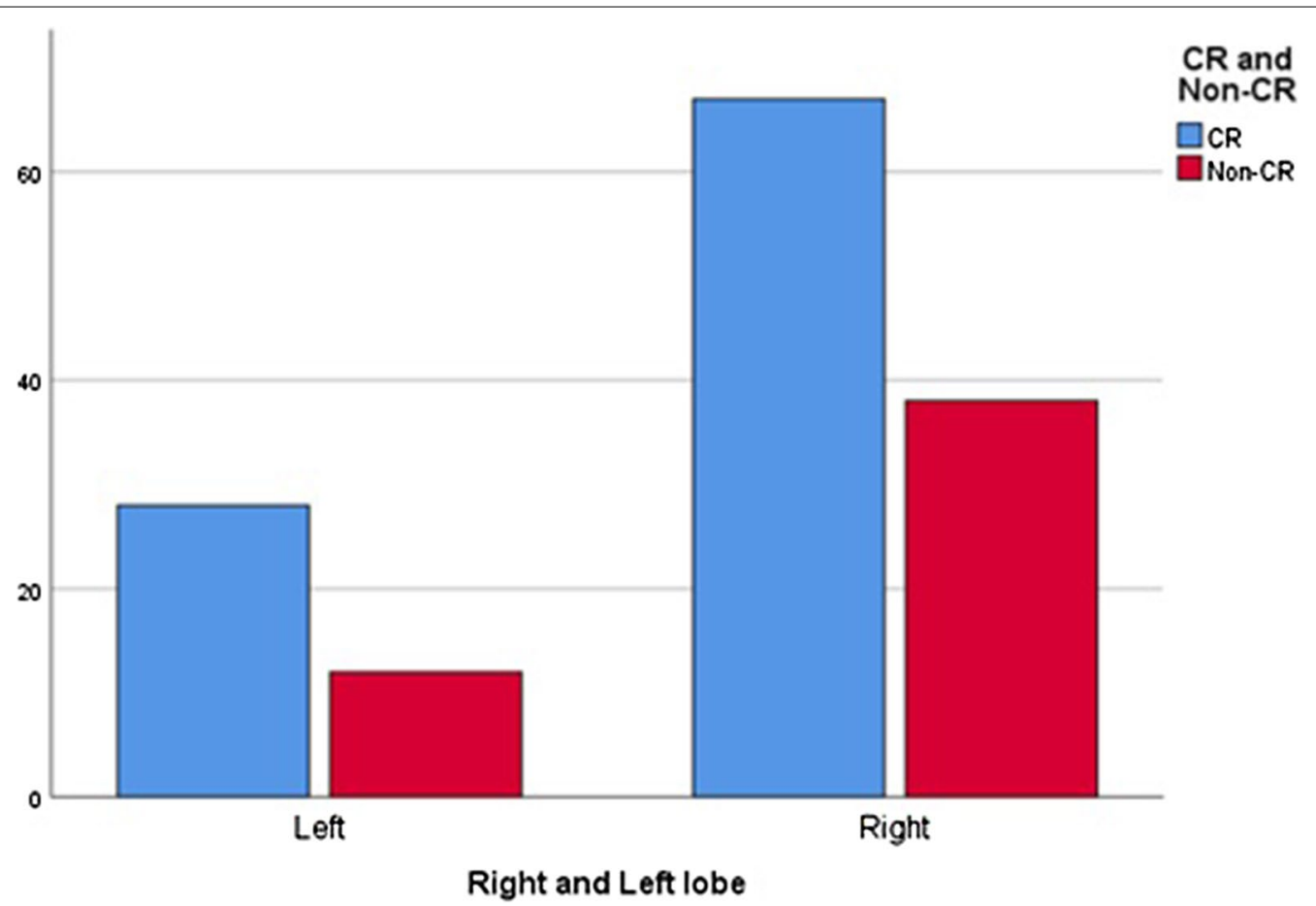

Fig. 5 Bar chart showing the association between the lobe of the tumor and CR and non-CR groups, no significant difference (P.48) 


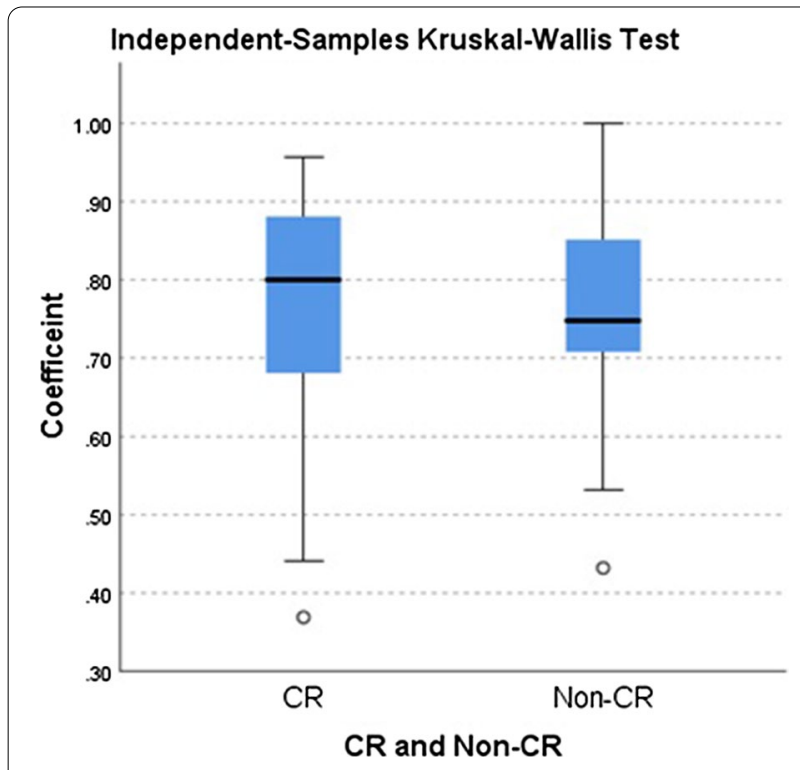

Fig. 6 Box plots of the relationship between central vs peripheral lesions and the $C R$ and non-CR groups, no significant difference ( $P$ .62)

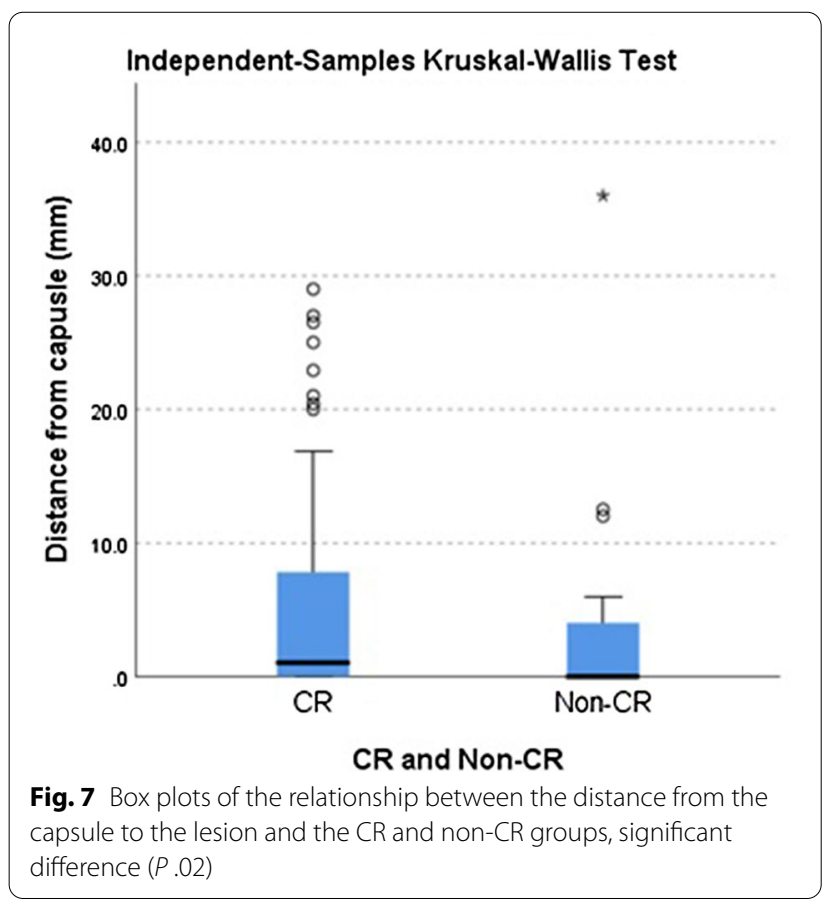

\section{Abbreviations}

TACE: Transarterial chemoembolization; HCC: Hepatocellular carcinoma; CR: Complete response; BCLC: Barcelona Clinic liver Cancer.

\section{Acknowledgements}

Not applicable

\section{Authors' contributions}

AE made the concept, design of the work, analysis and interpretation of the data. SMA has made analysis and interpretation of the data. HMN has drafted the work and substantively revised it. MSH made acquisition, analysis and interpretation of the data and substantively revised it. All authors have read and approved the manuscript.

\section{Funding}

No funding.

\section{Availability of data and materials}

The datasets used and/or analyzed during the current study are available from the corresponding author on reasonable request.

\section{Declarations}

\section{Ethics approval and consent to participate}

This study was approved by the Research Ethics Committee of the Faculty of Medicine at Ain Shams University in Egypt. The Committee's reference number is not applicable. Written informed consent was signed by all patients before the procedure.

\section{Consent for publication}

Consent for publication was obtained for every individual person's data included in the study.

\section{Competing interests}

The authors declare that they have no competing interests.

Received: 9 July 2021 Accepted: 6 September 2021

Published online: 14 September 2021

\section{References}

1. Sung H, Ferlay J, Siegel RL et al (2021) Global cancer statistics 2020: GLOBOCAN estimates of incidence and mortality worldwide for 36 cancers in 185 countries. CA Cancer J Clin 71(3):209-249. https://doi.org/10.3322/ caac. 21660

2. She WH, Cheung TT (2016) Bridging and downstaging therapy in patients suffering from hepatocellular carcinoma waiting on the list of liver transplantation. Transl Gastroenterol Hepatol 1:34. https://doi.org/10.21037/ $\operatorname{tgh} .2016 .03 .04$

3. Ozaki K, Matsui O, Kobayashi S, Minami T, Kitao A, Gabata T (2016) Morphometric changes in liver cirrhosis: aetiological differences correlated with progression. Br J Radiol 89(1059):20150896. https://doi.org/10.1259/ bjr.20150896

4. Ozaki K, Matsui O, Kobayashi S et al (2010) Selective atrophy of the middle hepatic venous drainage area in hepatitis C-related cirrhotic liver: morphometric study by using multidetector CT. Radiology 257(3):705714. https://doi.org/10.1148/radiol.10100468

5. Jang H-J, Khalili K, Yu H, Kim TK (2012) Perfusion and parenchymal changes related to vascular alterations of the liver. Abdom Imaging 37(3):404-421. https://doi.org/10.1007/s00261-011-9767-0

6. Lencioni R, Llovet JM (2010) Modified RECIST (mRECIST) assessment for hepatocellular carcinoma. Semin Liver Dis 30(1):52-60. https://doi.org/10. 1055/s-0030-1247132

7. EASL Clinical Practice Guidelines (2018) Management of hepatocellular carcinoma. J Hepatol 69(1):182-236. https://doi.org/10.1016/j.jhep.2018 03.019

8. Lladó L, Virgili J, Figueras J et al (2000) A prognostic index of the survival of patients with unresectable hepatocellular carcinoma after transcatheter arterial chemoembolization. Cancer 88(1):50-57. https://doi.org/10. 1002/(sici)1097-0142(20000101)88:1\%3c50::aid-cncr8\%3e3.0.co:2-i

9. Chan SL, MokT, Ma BBY (2012) Management of hepatocellular carcinoma: beyond sorafenib. Curr Oncol Rep 14(3):257-266. https://doi.org/10. 1007/s11912-012-0233-0

10. Miki I, Murata S, Uchiyama F et al (2017) Evaluation of the relationship between hepatocellular carcinoma location and transarterial 
chemoembolization efficacy. World J Gastroenterol 23(35):6437-6447. https://doi.org/10.3748/wjg.v23.i35.6437

11. Kim H-C, Chung JW, Lee W, Jae HJ, Park JH. Recognizing Extrahepatic Collateral Vessels That Supply Hepatocellular Carcinoma to Avoid Complications of Transcatheter Arterial Chemoembolization. RadioGraphics. 2005;25(suppl_1):S25-S39. doi:https://doi.org/10.1148/rg.25si055508

\section{Publisher's Note}

Springer Nature remains neutral with regard to jurisdictional claims in published maps and institutional affiliations.
Submit your manuscript to a SpringerOpen ${ }^{0}$ journal and benefit from:

- Convenient online submission

- Rigorous peer review

- Open access: articles freely available online

- High visibility within the field

- Retaining the copyright to your article

Submit your next manuscript at $\boldsymbol{\nabla}$ springeropen.com 